\title{
On the existence of soliton solutions for systems with a polynomial potential and their numerical realization*
}

\author{
L. A. Beklaryan ${ }^{1}$, and A. L. Beklaryan ${ }^{2,1}$ \\ beklar@cemi.rssi.ru; abeklaryan@hse.ru \\ ${ }^{1}$ Central Economics and Mathematics Institute RAS, Nachimovky prospect 47, 117418 Moscow; \\ ${ }^{2}$ National Research University Higher School of Economics, Kirpichnaya Ulitsa 33, 105187 Moscow
}

The problem of existence of soliton solutions (solutions of the traveling wave type) for the Korteweg-de Vries equation with a polynomial potential is considered on the basis of the approach within which the presence of a one-to-one correspondence of such solutions with solutions of the induced functional differential equation of pointwise type is demonstrated. On this path, conditions for the existence and uniqueness of solutions of the traveling wave type, with the growth restrictions both in time and in space, arise. It is very important that the conditions for the existence of a traveling wave solution are formed in terms of the right-hand side of the equation and the characteristics of the traveling wave, without using either the linearization and spectral properties of the corresponding equation in variations. Conditions for the existence of periodic soliton solutions are considered separately, and the possibility of transition from systems with a quasilinear potential to systems with a polynomial potential with conservation of corresponding existence theorems is demonstrated. Numerical implementation of such solutions is given.

Keywords: functional-differential equations; soliton solutions; Korteweg-de Vries equation; polynomial potential; periodic solitons

DOI: $10.21469 / 22233792.4 .4 .01$

\section{Introduction}

For equations of mathematical physics, which are the Euler-Lagrange equation of the corresponding variational problems, an important class of solutions are soliton solutions $[1,2]$. In a number of models, such solutions are well approximated by soliton solutions for finite-difference analogues of the initial equations, which, in place of a continuous environment, describe the interaction of clumps of a environment placed at the vertices of the lattice $[1,3]$. Emerging systems belong to the class of infinite-dimensional dynamical systems. The most widely considered classes of such problems are infinite systems with Frenkel-Kontorova potentials (periodic and slowly growing potentials) and Fermi-Pasta-Ulam (potentials of exponential growth), a broad survey of which is given in the paper [4].

The study of soliton solutions (solutions of the traveling wave) is based on the existence of a one-to-one correspondence between soliton solutions for infinite-dimensional dynamical systems and solutions of induced functional differential equations of pointwise type (FDEPT) [5] (in case of the quasilinear right-hand side of the functional differential equation). In fact, the described connection between solutions of the traveling wave type of the infinite-dimensional dynamical system and solutions of the induced FDEPT is a fragment of a more general scheme that goes beyond the scope of this article. Periodic soliton solutions of an infinite-dimensional dynamical system form an important class of solutions for both quasilinear and polynomial potentials.

\footnotetext{
*The reported study was partially supported by Russian Science Foundation, Project 17-71-10116. Also this work was partially funded by RFBR according to the research project 16-01-00110 A.
} 
It is important that the investigation of soliton solutions of an infinite-dimensional dynamical system is equivalent to the investigation of solutions of an induced FDEPT.

Another class of problems is related to the study of soliton solutions for equations of mathematical physics without using finitedifference analogs. At the same time, the potentials can have lags in time. In this case, the induced equation for traveling waves also turns out to be a FDEPT.

For both classes of problems, the existence and uniqueness theorem for a solution of an induced FDEPT [5] guarantees the existence and uniqueness of a soliton solution with given initial values for systems with quasilinear potential. Moreover, for systems with a quasilinear potential, one can formulate the conditions for the existence of a periodic solution. The formulated conditions, as well as the estimate of the radius of the ball in which the solution is contained, are given in terms of the characteristics of the right-hand side of the induced FDEPT: the Lipschitz constant, the deviation of the argument, and the characteristic of the traveling wave. It is very important that such conditions do not use information about the spectral properties of the linearized equation (equations in variations) that essentially simplifies their verification. A system with a polynomial potential can be redefined by changing the potential outside a given ball, so that the emerging potential turns out to be quasilinear. If a guaranteed periodic soliton solution for such an overdetermined system lies in the ball outside which the potential is redefined, then we obtain the conditions for the existence of a periodic soliton solution for the initial system with a polynomial potential. Another important task is the numerical realization of periodic soliton solutions for systems with a polynomial potential, which has been successfully solved.

\section{Functional differential equations of pointwise type}

The theory of functional differential equations developed in the works of many authors, among which it is necessary to single out the works of Myshkis, Bellman, Kato, Krasovsky, Krasnoselsky, Sharkovsky, Hale, Varga, and others. We consider a functional differential equation of pointwise type

$$
\dot{x}(t)=f\left(t, x\left(q_{1}(t), \ldots, x\left(q_{s}(t)\right)\right), \quad t \in B_{R},\right.
$$

where $f: \mathbb{R} \times \mathbb{R}^{n s} \longrightarrow \mathbb{R}^{n}$ — mapping of the $C^{(0)}$ class; $q_{j}(\cdot), j=1, \ldots, s$ - homeomorphisms of the line preserving orientation; $B_{R}$ is either closed interval $\left[t_{0}, t_{1}\right]$ or closed half-line $\left[t_{0},+\infty[\right.$ or line $\mathbb{R}$.

The FDEPT under consideration:

- is an ordinary differential equation if $q_{j}(t) \equiv t, j=1, \ldots, s$;

- is an equation with pure delays if $q_{j}(t) \leqslant t, j=1, \ldots, s$;

- is an equation with pure advances if $q_{j}(t) \geqslant t, j=1, \ldots, s$.

The functions $\left[q_{j}(t)-t\right], j=1, \ldots, s$ are called deviations of the argument. Using time replacement, we can always achieve the condition

$$
h=\max _{i \in\{1, \ldots, s\}} h_{j}<+\infty, \quad h_{j}=\sup _{t \in \mathbb{R}}\left|q_{j}(t)-t\right|, \quad j=1, \ldots, s
$$

for deviations of the argument. It is obvious that such a replacement of time can change the growth character of the right-hand side of the equation with respect to the time variable.

The approach proposed for the study of such equations is based on a formalism whose central element is the construction using a finitely generated group

$$
Q=<q_{1}, \ldots, q_{s}>
$$


of homeomorphisms of the line (the group operation in such a group is the superposition of two homeomorphisms). The type of functional-differential equations considered here is rather wide and, in particular, describes traveling-wave solutions (soliton solutions) for finite-difference analogues of the equations of mathematical physics. At the same time, the use of the specifics of such a class of equations related to group features makes it possible to obtain advanced results for them and also to establish obstacles that prevent such equations from inheriting the remarkable properties of solutions of ordinary differential equations.

Definition 1. An absolutely continuous function $x(\cdot)$ defined on $\mathbb{R}$ is called a solution of the equation (1) if for almost all $t \in B_{R}$ the function $x(\cdot)$ satisfies this equation. If, in addition, $x(\cdot) \in C^{(k)}\left(\mathbb{R}, \mathbb{R}^{n}\right), k=0,1, \ldots$ then this solution is called a solution of the class $C^{(k)}$.

\subsection{Existence and uniqueness of a solution for the initial-boundary value problem}

The main goal in the study of such differential equations is the investigation of the initialboundary value problem

$$
\begin{aligned}
& \dot{x}(t)=f\left(t, x\left(q_{1}(t), \ldots, x\left(q_{s}(t)\right)\right), \quad t \in B_{R},\right. \\
& \dot{x}(t)=\varphi(t), \quad t \in \mathbb{R} \backslash B_{R}, \quad \varphi(\cdot) \in L_{\infty}\left(\mathbb{R}, \mathbb{R}^{n}\right), \\
& x(\bar{t})=\bar{x}, \quad \bar{t} \in \mathbb{R}, \quad \bar{x} \in \mathbb{R}^{n},
\end{aligned}
$$

which we will call the basic initial-boundary value problem. In a general situation, when $\bar{t} \neq$ $=t_{0}, t_{1}$, or deviations of the argument are arbitrary, we have a problem with non-local initialboundary conditions.

If there is no deviation of the argument $\left(q_{j}(t) \equiv t, j=1, \ldots, s\right)$, the boundary value problem becomes the Cauchy problem for the ordinary differential equation.

If $B_{R}=\left[t_{0}, t_{1}\right]$ or $B_{R}=\left[t_{0},+\infty\left[\right.\right.$, then for $\bar{t}=t_{0}$ and for an equation with delays $\left(q_{j}(t) \leqslant\right.$ $t, j=1, \ldots, s)$, or for $\bar{t}=t_{1}$ and for an equation with advances $\left(q_{j}(t) \geqslant t, j=1, \ldots, s\right)$, the boundary value problem is transformed into the well-known formulation of the initial problem for an equation with delays or advances in the argument. It is important that in the noted cases, the problem under consideration has initial-boundary conditions of local type.

Let's define a Banach space of functions $x(\cdot)$ with weights

$$
\mathcal{L}_{\mu}^{n} C^{(k)}(\mathbb{R})=\left\{x(\cdot): x(\cdot) \in C^{(k)}\left(\mathbb{R}, \mathbb{R}^{n}\right), \max _{0 \leqslant r \leqslant k} \sup _{t \in \mathbb{R}}\left\|x^{(r)}(t) \mu^{|t|}\right\|_{\mathbb{R}^{n}}<+\infty\right\}, \quad \mu \in(0,1),
$$

with a norm

$$
\|x(\cdot)\|_{\mu}^{(k)}=\max _{0 \leqslant r \leqslant k} \sup _{t \in \mathbb{R}}\left\|x^{(r)}(t) \mu^{|t|}\right\|_{\mathbb{R}^{n}} .
$$

Let's formulate a system of restrictions on the right-hand side of FDEPT:

(a) $f(\cdot) \in C^{(0)}\left(\mathbb{R} \times \mathbb{R}^{n \times s}, \mathbb{R}^{n}\right)$ (function $f(\cdot)$ with respect to the variable $t$ can be considered as piecewise continuous function with discontinuities of the first kind at the points of a discrete set);

(b) quasilinear growth condition: for any $t, z_{j}, \bar{z}_{j}, j=1, \ldots, s$

$$
\left\|f\left(t, z_{1}, \ldots, z_{s}\right)\right\|_{\mathbb{R}^{n}} \leqslant M_{0}(t)+M_{1} \sum_{j=1}^{s}\left\|z_{j}\right\|_{\mathbb{R}^{n}}, \quad M_{0}(\cdot) \in C^{(0)}(\mathbb{R}, \mathbb{R})
$$

and Lipschitz condition

$$
\left\|f\left(t, z_{1}, \ldots, z_{s}\right)-f\left(t, \bar{z}_{1}, \ldots, \bar{z}_{s}\right)\right\|_{\mathbb{R}^{n}} \leqslant M_{2} \sum_{j=1}^{s}\left\|z_{j}-\bar{z}_{j}\right\|_{\mathbb{R}^{n}}
$$


(in fact $M_{1} \leqslant M_{2}$, but the constants $M_{1}$ and $M_{2}$ can be taken equal);

(c) there exists $\mu^{*} \in \mathbb{R}_{+}$such that the expression

$$
\sup _{i \in \mathbb{Z}} M_{0}(t+i)\left(\mu^{*}\right)^{|i|}
$$

has a finite value for any $t \in \mathbb{R}$ and is continuous as a function of the argument $t$.

(d) for the $\mu^{*}$ from the item (c) the family of functions

$$
\tilde{f}_{i, z_{1}, \ldots, z_{s}}(t)=f\left(t+i, z_{1}, \ldots, z_{s}\right)\left(\mu^{*}\right)^{|i|}, \quad i \in \mathbb{Z}, \quad z_{1}, \ldots, z_{s} \in \mathbb{R}^{n}
$$

is equicontinuous on any finite interval.

In the case of the finite interval of the definition $B_{R}=\left[t_{0}, t_{1}\right]$, we assume that the function $f(\cdot)$ satisfies the conditions $(a)-(b)$. If $B_{R}$ is a half-line or a whole line we assume that $f(\cdot)$ satisfies the conditions $(a)-(d)$. The last condition $(d)$ is necessary only in the case of a half-line or a whole line. It can be removed, but this leads to more technical complications.

Let's describe a very wide class of functions $f(\cdot)$ satisfying the constraints $(a)-(d)$.

Remark 1. Suppose that the function $f$ has a representation

$$
f\left(t, z_{1}, \ldots, z_{s}\right)=f_{1}\left(z_{1}, \ldots, z_{s}\right)+\xi(t)
$$

in which the continuous function $\xi(\cdot)$ satisfies the condition $(d)$, and the function $f_{1}$ satisfies the Lipschitz condition. Then the function $f$ satisfies the conditions $(a)-(d)$.

The right-hand side $f(\cdot)$ of FDEPT will be considered as an element of the Banach space $V_{\mu^{*}}\left(\mathbb{R} \times \mathbb{R}^{n s}, \mathbb{R}^{n}\right)$

$$
\begin{array}{r}
V_{\mu^{*}}\left(\mathbb{R} \times \mathbb{R}^{n s}, \mathbb{R}^{n}\right)=\{f(\cdot): f(\cdot) \text { satisfies the conditions }(a)-(d)\} \\
\|f(\cdot)\|_{L i p}=\sup _{t \in \mathbb{R}}\left\|f(t, 0, \ldots, 0)\left(\mu^{*}\right)^{|t|}\right\|_{\mathbb{R}^{n}}+ \\
+\sup _{\left(t, z_{1}, \ldots, z_{s}, \bar{z}_{1}, \ldots, \bar{z}_{s}\right) \in \mathbb{R}^{1+2 n s}} \frac{\left\|f\left(t, z_{1}, \ldots, z_{s}\right)-f\left(t, \bar{z}_{1}, \ldots, \bar{z}_{s}\right)\right\|_{\mathbb{R}^{n}}}{\sum_{j=1}^{s}\left\|z_{j}-\bar{z}_{j}\right\|_{\mathbb{R}^{n}}}
\end{array}
$$

where the parameter $\mu^{*} \in \mathbb{R}_{+}$coincides with the corresponding constant from the condition $(c)$.

Obviously, for the function $f(\cdot) \in V_{\mu^{*}}\left(\mathbb{R} \times \mathbb{R}^{n s}, \mathbb{R}^{n}\right)$ the smallest value of the constant $M_{2}$ from the Lipschitz condition (the condition $(b)$ ) coincides with the value of the second summand in the definition of the norm $f(\cdot)$. In what follows, speaking of the Lipschitz condition, by the constant $M_{2}$ we mean exactly its smallest value.

We have the following theorem on the existence and uniqueness of a solution.

Theorem 1 ( [6]). If for some $\mu \in\left(0, \mu^{*}\right) \cap(0,1)$ the inequality

$$
M_{2} \sum_{j=1}^{s} \mu^{-\left|h_{j}\right|}<\ln \mu^{-1},
$$

is satisfied then for any fixed initial-boundary conditions

$$
\varphi(\cdot) \in L_{\infty}\left(\mathbb{R}, \mathbb{R}^{n}\right), \quad \bar{x} \in \mathbb{R}^{n}
$$


there exists a solution (absolutely continuous)

$$
x(\cdot) \in \mathcal{L}_{\mu}^{n} C^{(0)}(\mathbb{R})
$$

of the basic initial-boundary value problem (2)-(4). Such a solution is unique and, as an element of the space $\mathcal{L}_{\mu}^{n} C^{(0)}(\mathbb{R})$, depends continuously on the initial-boundary conditions $\varphi(\cdot$ $\cdot) \in L_{\infty}\left(\mathbb{R}, \mathbb{R}^{n}\right), \bar{x} \in \mathbb{R}^{n}$ and the right-hand side of the equation (function $f(\cdot) \in V_{\mu^{*}}(\mathbb{R} \times$ $\left.\times \mathbb{R}^{n s}, \mathbb{R}^{n}\right)$ ).

\subsection{Existence of a periodic solution}

To simplify the presentation of the idea and the basic constructions of the proposed approach, we restrict ourselves to the case where the homeomorphisms $q_{j}, j=1, \ldots, s$ are shifts, i.e. $q_{j}(t)=t+n_{j}, n_{j} \in \mathbb{R}, j=1, \ldots, s$. Without loss of generality, we assume that $n_{1} \leqslant \ldots \leqslant n_{s}$. For clarity in the presentation, we confine ourselves to the case when the deviation values of the argument $n_{1}, \ldots, n_{s}$ and the period $\omega$ of the right-hand side of the equation with respect to the time variable are commensurable. $\mathrm{n}$ this case, without loss of generality, we can assume that the quantities $n_{1}, \ldots, n_{s}, \omega$ are integers. The last property can be achieved by replacing the time with extension.

We consider periodic solutions of the equation

$$
\dot{x}(t)=f\left(t, x\left(t+n_{1}\right), \ldots, x\left(t+n_{s}\right)\right), \quad t \in \mathbb{R}
$$

with a $\omega$ time-periodic right-hand side. Let's formulate an existence theorem for a periodic solution in terms of averages over the period.

Theorem 2 ( $[7])$. Suppose that the map $f(\cdot)$ satisfies the conditions $(a)-(d)$ and is a $\omega$-periodic function with respect to time, where $\omega \in \mathbb{Z}_{+}$. If for given $\mu \in\left(0, \mu^{*}\right) \cap(0,1), r>0$ and for all $\bar{x} \in \mathbb{R}^{n},\|\bar{x}\|_{\mathbb{R}^{n}}=r$ it is true that

$$
\begin{gathered}
M_{2} \sum_{j=1}^{s} \mu^{-\left|n_{j}\right|}<\ln \mu^{-1}, \\
\left(\frac{\bar{x}}{\|\bar{x}\|_{\mathbb{R} n}^{n}}, \int_{0}^{\omega} f(\tau, \bar{x}, \ldots, \bar{x}) d \tau\right)<-\frac{\left(\mu^{-\omega}-1\right) M_{2}\left(\sum_{j=1}^{s} \mu^{-\left|n_{j}\right|}\right)}{\ln \mu^{-1}\left(\ln \mu^{-1}-M_{2} \sum_{j=1}^{s} \mu^{-\left|n_{j}\right|}\right)} \times \\
\times\left[M_{2} s r+\inf _{\xi \in[0, \omega]} \sup _{\tau \in[\xi, \xi+1]} M_{0 \infty \mu}(\tau)\right],
\end{gathered}
$$

then for the initial FDEPT (5) there exists $\omega$-periodic solution $x(\cdot),\|x(0)\|_{\mathbb{R}^{n}} \leqslant r$, which lies in the ball of the space $\mathbb{R}^{n}$ with radius $\mu^{-\omega} \hat{\mathcal{R}}$, where

$$
\begin{gathered}
\hat{\mathcal{R}}=r+\frac{\left[M_{2} s r+\inf _{\xi \in[0, \omega]} \sup _{\tau \in[\xi, \xi+1]} M_{0 \infty \mu}(\tau)\right]}{\left(\ln \mu^{-1}-M_{2} \sum_{j=1}^{s} \mu^{-\left|n_{j}\right|}\right)}, \\
M_{0 \infty \mu}(t)=\sup _{i \in \mathbb{Z}} M_{0}(t+i) \mu^{|i|} .
\end{gathered}
$$

\subsection{Approximation of the solution of a functional differential equation defined on the line by solutions of a initial-boundary value problem with expanding intervals of the definition}

On the basis of the Theorem 1 we are going to formulate a proposition on the approximation of solutions of an initial-boundary value problem defined on the whole line by solutions of the 
initial-boundary value problem defined on the interval $[-k, k]$ as $k \rightarrow+\infty$. We consider the initial-boundary value problem on the whole line $B_{R}=\mathbb{R}$

$$
\begin{aligned}
& \dot{x}(t)=f\left(t, x\left(t+n_{1}\right), \ldots, x\left(t+n_{s}\right)\right), \quad t \in \mathbb{R}, \\
& x(\bar{t})=\bar{x}, \quad \bar{t} \in \mathbb{R}, \quad \bar{x} \in \mathbb{R}^{n}
\end{aligned}
$$

and for each $k \in \mathbb{Z}$ the initial-boundary value problem on a finite interval $B_{R}=[-k, k]$

$$
\begin{aligned}
& \dot{x}(t)=f\left(t, x\left(t+n_{1}\right), \ldots, x\left(t+n_{s}\right)\right), \quad t \in[-k, k], \\
& \dot{x}(t)=\varphi(t), \quad t \in \mathbb{R} \backslash[-k, k], \quad \varphi(\cdot) \in \mathcal{L}_{1}^{n} L_{\infty}(\mathbb{R}), \\
& x(\bar{t})=\bar{x}, \quad \bar{t} \in \mathbb{R}, \quad \bar{x} \in \mathbb{R}^{n} .
\end{aligned}
$$

Theorem 3 ( [8]). If for $\mu \in\left(0, \mu^{\star}\right) \cap(0,1)$ the inequality

$$
M_{2} \sum_{j=1}^{s} \mu^{-\left|n_{j}\right|}<\ln \mu^{-1},
$$

is satisfied, and $\left(\mu_{1}, \mu_{2}\right)$ is the maximal interval on which the inequality (11) holds, then for any $\bar{x} \in \mathbb{R}^{n}, \varphi(\cdot) \in \mathcal{L}_{1}^{n} L_{\infty}(\mathbb{R})$ the solution $\hat{x}(\cdot)$ of the initial-boundary value problem (6)-(7), as an element of the space $\mathcal{L}_{\mu}^{n} C^{(0)}(\mathbb{R})$, is approximated by solutions $\hat{x}_{k}(\cdot)$ of the initial-boundary value problem (8)-(10) as $k \rightarrow+\infty$ and for any arbitrarily small number $\varepsilon, 0<\varepsilon<\mu_{2}-\mu_{1}$ there is $C_{f, \varepsilon}$ such that the following estimate is true

$$
\left\|\hat{x}(\cdot)-\hat{x}_{k}(\cdot)\right\|_{\mu}^{(0)} \leqslant C_{f, \varepsilon}\left(\frac{\mu_{1}}{\mu_{2}-\varepsilon}\right)^{k} .
$$

We note that in the sequence of initial-boundary value problems on intervals $[-k, k]$ the boundary function can be an arbitrary fixed essentially bounded function $\varphi(\cdot) \in \mathcal{L}_{1}^{n} L_{\infty}(\mathbb{R})$.

\section{Soliton solutions of the Korteweg-de Vries equation with a polynomial potential}

There has been a particular interest in the theory of Korteweg-de Vries (KdV) equation due to its significance in nonlinear dispersive wave theory. Many different real-world nonlinear physical problems are modeled by this well-known equation $[9,10]$. For example, this equation has many direct physical applications to solids, liquids, gases, pedestrian flow models [11], car-following models $[12,13]$ and so on.

It has widely been argued and accepted $[14,15]$ that for various reasons, time delay should be taken into consideration in modeling. Zhao and $\mathrm{Xu}$ [16] has considered solitary wave solutions of the KdV equation with delays, also Zhao dealt with the initial-value problem of the delay $\mathrm{KdV}$ equation [17]. Therefore, we want to incorporate a single discrete time delay $\tau>0$ into $\mathrm{KdV}$ equation and consider the delay KdV-type equation's initial-boundary value problem.

$\mathrm{KdV}$-type class of equations has different versions of the representation, but further we will concentrate on finding numerical solutions for the equation of the following form

$$
\begin{aligned}
& \ddot{x}(t)=c[\alpha x(t)+(1-\alpha) x(t-\tau)]+\frac{1}{2} x^{2}(t)+\tau \dot{x}(t-\tau), \\
& t \in \mathbb{R}, \quad \alpha \in[0,1], \quad c, \tau \in \mathbb{R}_{+} .
\end{aligned}
$$


It should also be noted that following [16], the second-order FDEPT (12) can be obtained by integrating an equation after substitution a solitary wave solution $U(x, t)=\varphi(x+c t)$ to the $\mathrm{KdV}$ equation with time delay

$$
\left[\alpha U_{t}(x, t)+(1-\alpha) U_{t}(x, t-\tau)\right]+U(x, t) U_{x}(x, t)+\tau U_{x x}(x, t-\tau)-U_{x x x}(x, t)=0 .
$$

In the previous sections, there was noted a one-to-one correspondence between solutions of traveling wave type for infinite-dimensional systems of ordinary differential equations and solutions of induced FDEPT. In particular, such infinite-dimensional differential equations can arise as finite-difference analogues of continuous systems. Here we consider traveling waves for the KdV equation as a continuous system. Moreover, we consider KdV equation with a delay. The arising equations, satisfied by traveling waves, are also FDEPT. For such equations with a quasilinear right-hand side, conditions for the existence of periodic solutions were obtained [7], as well as an estimate of the radius of the ball in which such solutions change. For equations of traveling waves with a polynomial right-hand side, redefining the right-hand side outside a certain sphere, one can achieve that it becomes quasilinear. Applying the corresponding result on the existence of a periodic solution for equations with a quasilinear right-hand side, one can obtain periodic solutions for the initial equation of traveling waves, and also a description of the range of parameters under which this periodicity takes place. Below, we demonstrate how the algorithm for constructing a numerical solution, following the logic of the correspondences noted above, allows efficient construction of traveling wave solutions.

\section{Numerical realization of periodic soliton solutions of the Korteweg-de Vries equation with a polynomial potential}

\subsection{Optimization problem and numerical methods}

Let's give a formal statement of the optimization problem for the search for numerical solutions of FDEPT. We consider the system

$$
F_{i}\left(t, \dot{x}(t), x\left(t+n_{1}\right), \ldots, x\left(t+n_{s}\right)\right)=0, \quad i=\overline{1, k}, \quad t \in\left[t_{l}, t_{r}\right],
$$

where $F: \mathbb{R} \times \mathbb{R}^{n} \times \mathbb{R}^{n s} \longrightarrow \mathbb{R}^{n}$ - mapping of $C^{(0)}$ class; $n_{j} \in \mathbb{Z}, j=\overline{1, s} ; t_{l}, t_{r} \in \mathbb{R}$; the values of the derivatives of the phase variables are defined on the extended interval $t \in\left[t_{l l}, t_{r r}\right]$, $t_{l l}=t_{l}+\min \left\{0, n_{1}, \ldots, n_{s}\right\}, t_{r r}=t_{r}+\max \left\{0, n_{1}, \ldots, n_{s}\right\}$

$$
\begin{aligned}
& \dot{x}(t)=h_{l}(t), \quad t \in\left[t_{l l}, t_{l}\right], \\
& \dot{x}(t)=h_{r}(t), \quad t \in\left[t_{r}, t_{r r}\right],
\end{aligned}
$$

where $h_{l}, h_{r}: \mathbb{R}^{n} \longrightarrow \mathbb{R}^{n}$ - mapping of $C^{(0)}$ class. The initial-boundary conditions are given by the functionals

$$
K_{m}\left(\dot{x}(\tau), x\left(\tau_{1}\right), \ldots, x\left(\tau_{p}\right)\right)=0, \quad m=\overline{1, q}, \quad \tau, \tau_{i} \in\left[t_{l}, t_{r}\right], \quad i=\overline{1, p} .
$$

The optimization problem consists in finding a trajectory $\hat{x}(t)$ that delivers the minimum of the residual functional $(\mathrm{RF})$

$$
\begin{aligned}
& I(\hat{x}(t))=v^{(N)}\left(\sum_{i=1}^{k} \int_{t_{l}}^{t_{r}} F_{i}^{2}\left(t, \dot{\hat{x}}(t), \hat{x}\left(t+n_{1}\right), \ldots, \hat{x}\left(t+n_{s}\right)\right) d t+\right. \\
& \left.\int_{t_{l l}}^{t_{l}}\left[\dot{\hat{x}}(t)-h_{l}(t)\right]^{2} d t+\int_{t_{r}}^{t_{r r}}\left[\dot{\hat{x}}(t)-h_{r}(t)\right]^{2} d t\right)+ \\
& v^{(K)} \sum_{m=1}^{q} K_{m}^{2}\left(\dot{\hat{x}}(\tau), \hat{x}\left(\tau_{1}\right), \ldots, \hat{x}\left(\tau_{p}\right)\right),
\end{aligned}
$$


where $v^{(N)}, v^{(K)} \in \mathbb{R}_{+}$- weighting coefficients.

The proposed approach to the investigation of boundary value problems is based on the Ritz method and spline-collocation constructions and was implemented in [18-20]. To solve the problems of the class under consideration, the trajectories of the system are discretized on a grid with a constant step, and a generalized residual functional is formulated that includes both the weighted residual of the original differential equation and the residual of the boundary conditions. To evaluate the derivatives of the desired trajectories of the system, a spline differentiation technique is used, based on two spline approximation designs: using cubic natural splines and using a special type of spline whose second derivatives at the edges are also controlled using optimized parameters.

For solving the stated finite-dimensional problems, a set of algorithms for local optimization (quasi-Newtonian method BFGS; two versions of the Powell's method; Barzilai-Borwein method; version of the method of confidence domains; stochastic search methods in random subspaces of dimension 3, 4, and 5, and others) and global optimization (method of random multistart; method of curvilinear searching; tunnel method; parabolic method, and others) was implemented. The used technology includes: an algorithm for sequentially increasing the accuracy of approximation by multiplying the number of nodes in the grid of the discretization; the algorithms for the difference evaluation of the derivatives of the functional - from the first to the sixth degree of accuracy inclusive; the method of successively increasing the precision of spline differentiation.

The corresponding software complex (SC) OPTCON-F was implemented in the language $C$ under the control of operating systems $O S$ Windows, OS Linux and Mac OS using compilers $B C C 5.5$ and $G C C$. SC was designed to obtain a numerical solution of boundary value problems, parametric identification problems and optimal control for dynamical systems described by FDEPT [21].

In the SC OPTCON-F there is the possibility of sequential application of various algorithms within the framework of constructing a solution for one task. Thus, the constructed intermediate solution in the previous step becomes the starting solution ("baseline") for the following algorithm. In this case, such an implementation does not prevent the global search algorithms from "popping out" of the local solution. Separately, we note the presence of a programming module that allows predetermining the order of application of algorithms, as well as the construction of complex chain of steps (conditional statements, cycles, etc.) depending on the current or historical values of a number parameters (for example, error estimation or number of iterations). For the examples presented below, the following scheme was used in cycle: the generalized quasi-Newtonian and Powell-Brent's methods (with bi-directional line search along each dimension) were used alternately, and after the error changed by less than $10^{-h}$ the adaptive modification of the Hooke-Jeeves method was used $l$ times $(h$ and $l$ are computable functions on the basis of the loop iteration number, as well as the Lipschitz constants of the equation itself and a number of other technical characteristics). The stopping criterion depended on the number of iterations in the first part of the cycle, as well as the current error estimate and its dynamics. In view of the above, as well as stochastic elements in the applied algorithms, the presented value of the residual functional ( $R F$, i.e. error of a solution) can not be used to estimate the theoretical rate of convergence.

The heuristic search algorithm for the solution $\hat{x}(t)$ can be justified on the basis of the existence and uniqueness theorems for initial-boundary value problems for the investigated FDEPT, as well as theorems on approximating solutions of such equations on the whole line 
by solutions of the initial-boundary value problem on a sequence of expanding intervals, the description of which was given in the previous sections.

\subsection{Statement of the numerical problem}

Next, the results of the computational experiments on the study of initial-boundary value problems for systems of FDEPT using OPTCON-F software will be presented. Let's consider the FDEPT of the following form

$$
\ddot{x}(t)=c[\alpha x(t)+(1-\alpha) x(t-c \tau)]+\frac{1}{2} x^{2}(t)+\tau \dot{x}(t-c \tau), \quad t \in \mathbb{R},
$$

where $\alpha \in[0,1], c, \tau \in \mathbb{R}_{+}$. Using a time-variable transformation the equation (13) can be rewritten in the form of the following system of equations of the first order

$$
\left\{\begin{array}{l}
\dot{z}_{1}(t)=c \tau z_{2}(t) \\
\dot{z}_{2}(t)=c \tau\left(c\left[\alpha z_{1}(t)+(1-\alpha) z_{1}(t-1)\right]+\frac{1}{2} z_{1}^{2}(t)+\tau z_{2}(t-1)\right)
\end{array}\right.
$$

Under this system, we have the following real parameters: $\alpha, c, \tau$. In the following example, for a given system, we consider such parameter values that conditions of the modified existence theorem for periodic solutions are fulfilled.

\subsection{Example}

We consider dynamical system in the following form:

$$
\begin{aligned}
& \left\{\begin{array}{l}
\dot{z}_{1}(t)=0.01 z_{2}(t) \\
\dot{z}_{2}(t)=0.01\left(0.1 z_{1}(t)+0.9 z_{1}(t-1)+\frac{1}{2} z_{1}^{2}(t)+0.01 z_{2}(t-1)\right),
\end{array}\right. \\
& \text { initial conditions } \\
& \left\{\begin{array}{l}
z_{1}(0)=-5 \\
z_{2}(0)=0
\end{array}\right.
\end{aligned}
$$

Here, with respect to the system (14), we have $\alpha=0.1, \tau=0.01, c=1$.

Taking into account the impossibility of considering the numerical solution of the system on an infinite interval, we introduce the parameter $k$ and the corresponding family of expanding initial-boundary value problems

$$
\left\{\begin{array}{l}
\dot{z}_{1}(t)=0.01 z_{2}(t) \\
\dot{z}_{2}(t)=0.01\left(0.1 z_{1}(t)+0.9 z_{1}(t-1)+\frac{1}{2} z_{1}^{2}(t)+0.01 z_{2}(t-1)\right),
\end{array} t \in[-k, k],\right.
$$

boundary conditions

$$
\left\{\begin{array}{l}
\dot{z}_{1}(t)=0, \\
\dot{z}_{2}(t)=0,
\end{array} \quad t \in(-\infty,-k] \cup[k,+\infty),\right.
$$

initial conditions

$$
\left\{\begin{array}{l}
z_{1}(0)=-5 \\
z_{2}(0)=0
\end{array}\right.
$$




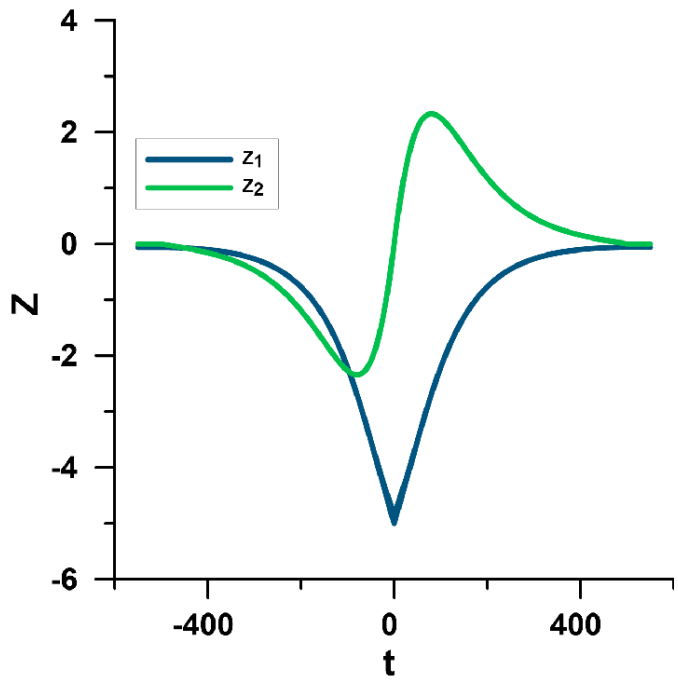

(a) $k=500, R F \simeq 3.1 \times 10^{-5}$

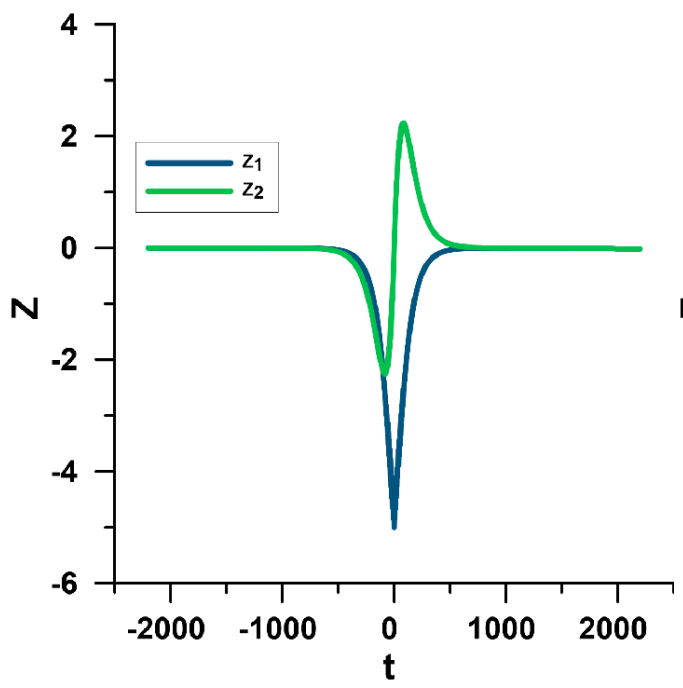

(в) $k=2000, R F \simeq 7.5 \times 10^{-6}$

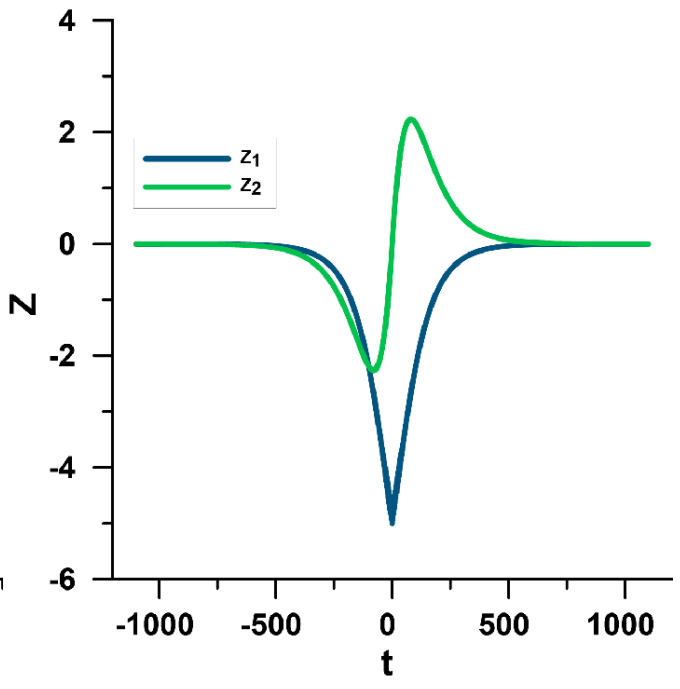

(б) $k=1000, R F \simeq 1.6 \times 10^{-5}$

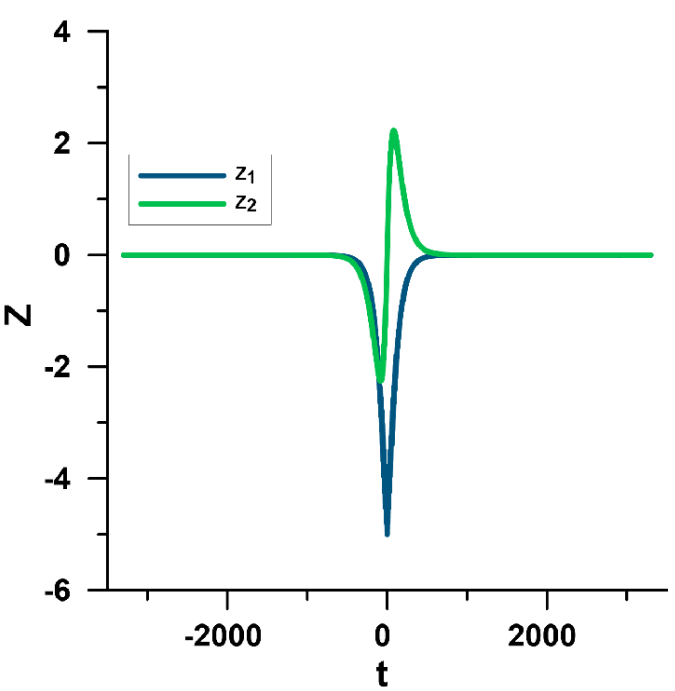

(2) $k=3000, R F \simeq 4.7 \times 10^{-6}$

Рис. 1 Trajectories of the system (16) at different $k$. 


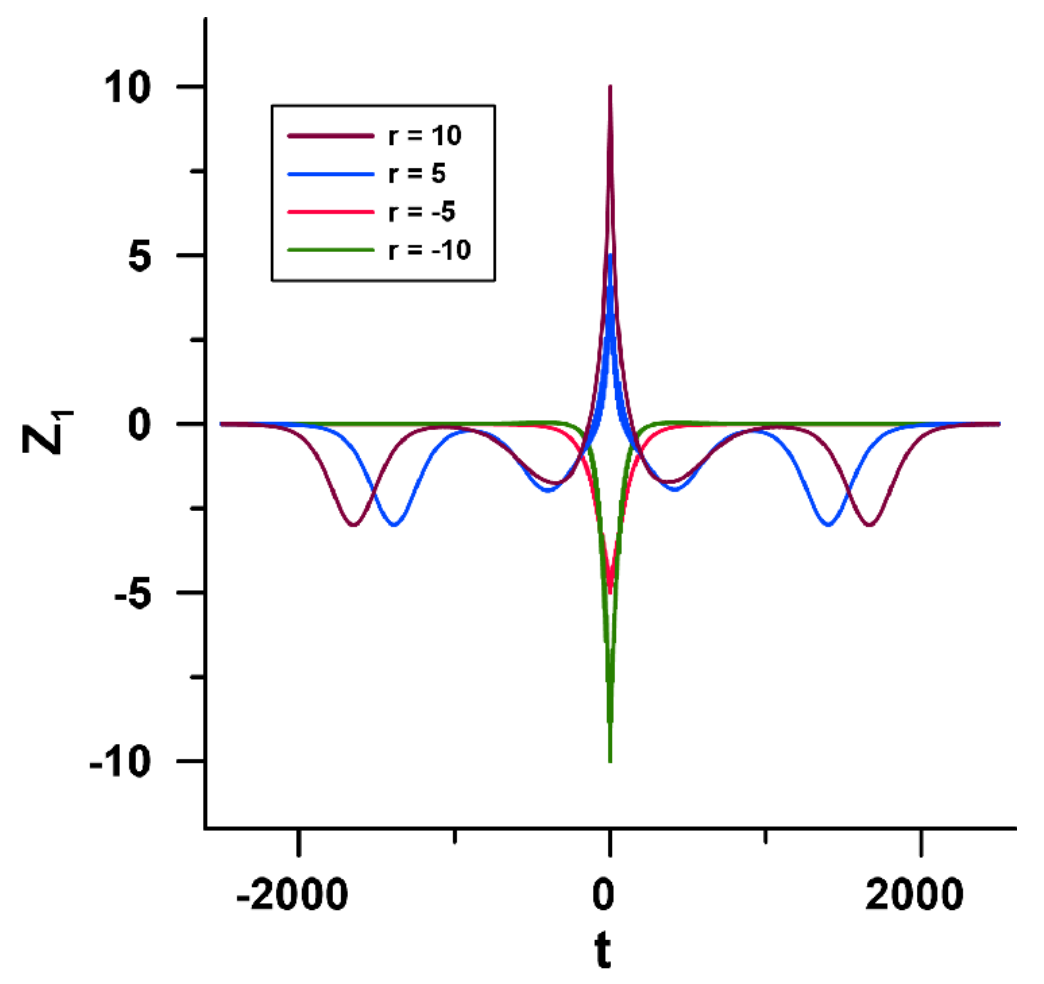

Figure 2 Trajectories of the system (16) at different $r$

According to the mentioned theoretical results, the solution of the system (16) converges to the solution of the system (15) as $k \rightarrow \infty$. The graphs of the solution of the system (16) at different values of $k$ are shown in Fig. 1.

Since the equation (13) is autonomous, the solution space of such equation is invariant with respect to time-variable shifts. Therefore, it suffices to consider a family of solutions of the initial problem (15) with a different values of $z_{1}(0)$. Figure 2 shows the integral curves for different values of the parameter $r=z_{1}(0)$ for the system (16). Note that for values of $|r|$ greater than about 20, "destruction" of traveling waves occurs and the residual functional of the numerical solution begins to grow rapidly, although for smaller values of $|r|$ it does not exceed $3 \times 10^{-4}$.

\section{Concluding Remarks}

On the example of the Korteweg-de Vries equation with a polynomial potential, an approach is realized in which the existence of a periodic soliton solution was established. Such an approach is based on redefinition of the right-hand side of the equation outside some given ball of phase space, under which the overdetermined potential turns out to be quasilinear. Conditions for the existence of a periodic soliton solution for an equation with an overdetermined quasilinear potential were formulated, which guarantee the condition for the embedding of such a solution in the noted ball of phase space. In such a case, such a solution turns out to be a periodic soliton solution for the initial equation with a polynomial potential. A numerical realization of such a periodic soliton solution was presented.

\section{References}

[1] Toda, M. 1989. Theory of Nonlinear Lattices. Springer, Berlin, Heidelberg. 225 p. 
[2] Miwa, T., M. Jimbo, and E. Date. 2000. Solitons: Differential Equations, Symmetries and Infinite Dimensional Algebras. Cambridge University Press. 122 p.

[3] Frenkel, Y. I., and T. A. Contorova. 1938. On the theory of plastic deformation and twinning. Journal of Experimental and Theoretical Physics 8(1):89-95.

[4] Pustyl'nikov, L. D. 1997. Infinite-dimensional non-linear ordinary differential equations and the kam theory. Russian Mathematical Surveys 52(3):551-604. doi: http://dx.doi.org/10.1070/ RM1997v052n03ABEH001810.

[5] Beklaryan, L. A. 2007. Vvedenie v teoriju funkcinal'no-differencial'nyh uravnenij. Gruppovoj podhod [Introduction to the theory of functional differential equations. Group approach]. Factorial Press, Moscow. 288 p. (In Russian).

[6] Beklaryan, L. A. 1991. A method for the regularization of boundary value problems for differential equations with deviating argument. Soviet Math. Dokl. 43:567-571.

[7] Beklaryan, L. A. 2018. A new approach to the issue of the existence of periodic solutions for functional differential equations of pointwise type. Izvestiya: Mathematics doi: http://dx.doi. org/10.1070/IM8662.

[8] Beklaryan, L. A., and A. L. Beklaryan. Approximation of solutions of functional differential equations of pointwise type by solutions of the induced optimization problem. Journal of Global Optimization (unpubl.).

[9] Gardner, C. S., J. M. Greene, and M. D. Kruskal. 1967. Method for solving the korteweg-de vries equation. Physical Review Letters 19(19):1095-1097. doi: http://dx.doi.org/10.1103/ PhysRevLett.19.1095.

[10] Kortweg, D. J., and G. De Vries. 1895. On the change of form of long waves advancing in a rectangular canal, and on a new type of long stationary waves. Philosophical Magazine 5(39):422443.

[11] Xu, L., S. Lo, and H. Ge. 2013. The korteweg-de vires equation for bidirectional pedestrian flow model. Procedia Engineering 52:495-499. doi: http://dx.doi.org/10.1016/j.proeng. 2013. 02.174 .

[12] Sun, D., D. Chen, M. Zhao, W. Liu, and L. Zheng. 2018. Linear stability and nonlinear analyses of traffic waves for the general nonlinear car-following model with multi-time delays. Physica A: Statistical Mechanics and its Applications 501:293-307. doi: http://dx.doi.org/10.1016/j. physa.2018.02.179.

[13] Yu, L., Z. Shi, and T. Li. 2014. A new car-following model with two delays. Physics Letters A 378(4):348-357. doi: http://dx.doi.org/10.1016/j.physleta.2013.11.030.

[14] Hale, J. K., and S. M. Verduyn Lunel. 1993. Introduction to Functional Differential Equations. Springer, New York. Vol. 99. 450 p.

[15] Wu, J. H. 1996. Theory and Applications of Partial Functional Differential Equations. SpringerVerlag, New York. Vol. 119. 432 p.

[16] Zhao, Z., and Y. Xu. 2010. Solitary waves for korteweg-de vries equation with small delay. Journal of Mathematical Analysis and Applications 368(1):43-53. doi: http://dx.doi.org/10. 1016/j . jmaa.2010.02.014.

[17] Zhao, Z., E. Rong, and X. Zhao. 2012. Existence for korteweg-de vries-type equation with delay. Advances in Difference Equations 2012(1): 64. doi: http://dx.doi.org/10.1186/ 1687-1847-2012-64.

[18] Zarodnyuk, T. S., A. S. Anikin, E. A. Finkelshtein, A. L. Beklaryan, and F. A. Belousov. 2016. Tehnologija reshenija kraevyh zadach dlja nelinejnyh sistem funkcional'no-differencial'nyh uravnenij tochechnogo tipa [the technology for solving the boundary value problems for nonlinear 
systems of functional differential equations of pointwise type]. Sovremennye tehnologii. Sistemnyj analiz. Modelirovanie [Modern technologies. System analysis. Modeling] 49(1):19-26. (In Russian).

[19] Zarodnyuk, T. S., A. Y. Gornov, A. S. Anikin, and E. A. Finkelstein. 2017. Computational technique for investigating boundary value problems for functional-differential equations of pointwise type. Proc. of the VIII International Conference on Optimization and Applications (OPTIMA-2017), Petrovac, Montenegro, October 2-\%. CEUR-WS.org. Available at: http://ceur-ws.org/Vol-1987/paper82.pdf.

[20] Beklaryan, L. A., and A. L. Beklaryan. 2017. Traveling waves and functional differential equations of pointwise type. what is common? Proc. of the VIII International Conference on Optimization and Applications (OPTIMA-2017), Petrovac, Montenegro, October 2-7. CEUR-WS.org. Available at: http://ceur-ws.org/Vol-1987/paper13.pdf.

[21] Gornov, A. Y., T. S. Zarodnyuk, T. I. Madzhara, A. V. Daneeva, and I. A. Veyalko. 2013. A collection of test multiextremal optimal control problems. Springer, New York, 257-274.

Received December 21, 2018

\section{$\mathrm{K}$ вопросу существования солитонных решений для систем с полиномиальным потенциалом и их численная реализация*}

\footnotetext{
Л. А. Бекларлн ${ }^{1}$, А.Л. Бекларян ${ }^{2,1}$

beklar@cemi.rssi.ru; abeklaryan@hse.ru

${ }^{1}$ Центральный Экономико-Математический Институт РАН, 117418 Москва, Нахимовский пр.47;

${ }^{2}$ Национальный исследовательский университет «Высшая школа экономики», 105187 Москва, ул. Кирпичная 33

В работе рассматривается вопрос существования солитонных решений (решений типа бегущей волны) для уравнения Кортевега - де Фриза с полиномиальным потенциалом базе подхода, в рамках которого показано наличие взаимно однозначного соответствия таких решений с решениями индуцированного функционально-дифференциального уравнения точечного типа. На этом пути возникают условия существования и единственности решений типа бегущей волны с условиями роста как по времени, так и по пространству (по координатам). Весьма важно, что условия существования решения типа бегущей волны формируются в терминах правой части уравнения и характеристики бегущей волны, не используя какие-либо линеаризации и спектральные свойства соответствующего уравнения в вариациях. Отдельно рассмотрены условия существования периодических солитонных решений, при этом продемонстрирована возможность перехода от систем с квазилинейным потенциалом к системам с полиномиальным потенциалом с сохранением соответствующих теорем существования. Приведена численная реализация подобных решений.
}

Ключевые слова: функиионально-дифференииалъные уравнения; солитонные решения; уравнение Кортевега - де Фриза; полиномиалъный потени,ил; периодические решения

DOI: $10.21469 / 22233792.4 .4 .01$

\footnotetext{
*Исследование частично выполнено за счет гранта Российского научного фонда (проект №17-71-10116), а также работа выполнена при частичной поддержке РФФИ (грант №16-01-00110 А).
} 


\section{Литература}

[1] Toda M. Theory of Nonlinear Lattices. - Berlin, Heidelberg: Springer, 1989. 225 p.

[2] Miwa T., Jimbo M., Date E. Solitons: Differential Equations, Symmetries and Infinite Dimensional Algebras. - UK: Cambridge University Press, 2000. 122 p.

[3] Frenkel Ya. I., Contorova T. A. On the theory of plastic deformation and twinning // Journal of Experimental and Theoretical Physics, 1938. Vol. 8. No. 1. P. 89-95.

[4] Pustyl'nikov L. D. Infinite-dimensional non-linear ordinary differential equations and the kam theory // Russian Mathematical Surveys, 1997. Vol. 52. No. 3. P. 551-604. doi: http://dx.doi. org/10.1070/RM1997v052n03ABEH001810.

[5] Бекларян Л. А. Введение в теорию функцинально-дифференциальных уравнений. Групповой подход. - Москва: Факториал Пресс, 2007. 288 с.

[6] Beklaryan L. A. A method for the regularization of boundary value problems for differential equations with deviating argument // Soviet Math. Dokl., 1991. Vol. 43. P. 567-571.

[7] Beklaryan L. A. A new approach to the issue of the existence of periodic solutions for functional differential equations of pointwise type // Izvestiya: Mathematics, 2018. Vol. 82. doi: http://dx. doi.org/10.1070/IM8662.

[8] Beklaryan L. A., Beklaryan A. L. Approximation of solutions of functional differential equations of pointwise type by solutions of the induced optimization problem // Journal of Global Optimization. (unpubl.).

[9] Gardner C. S., Greene J. M., Kruskal M. D. Method for solving the korteweg-de vries equation // Physical Review Letters, 1967. Vol. 19. No. 19. P. 1095-1097. doi: http://dx.doi.org/10.1103/ PhysRevLett.19.1095.

[10] Kortweg D. J., De Vries G. On the change of form of long waves advancing in a rectangular canal, and on a new type of long stationary waves // Philosophical Magazine, 1895. Vol. 5. No. 39. P. 422-443.

[11] Xu L., Lo S., Ge H. The korteweg-de vires equation for bidirectional pedestrian flow model // Procedia Engineering, 2013. Vol. 52. P. 495-499. doi: http://dx.doi.org/10.1016/j.proeng. 2013.02.174.

[12] Sun D., Chen D., Zhao M., Liu W., Zheng L. Linear stability and nonlinear analyses of traffic waves for the general nonlinear car-following model with multi-time delays // Physica A: Statistical Mechanics and its Applications, 2018. Vol. 501. P. 293-307. doi: http://dx.doi. org/10.1016/j.physa.2018.02.179.

[13] Yu L., Shi Z., Li T. A new car-following model with two delays // Physics Letters A, 2014 . Vol. 378. No. 4. P. 348-357. doi: http://dx.doi.org/10.1016/j.physleta.2013.11.030.

[14] Hale J. K., Verduyn Lunel S. M. Introduction to Functional Differential Equations. - New York: Springer, 1993. Vol. 99. 450 p.

[15] $W u$ J. H. Theory and Applications of Partial Functional Differential Equations. - New York: Springer-Verlag, 1996. Vol. 119. 432 p.

[16] Zhao Z., Xu Y. Solitary waves for korteweg-de vries equation with small delay // Journal of Mathematical Analysis and Applications, 2010. Vol. 368. No. 1. P. 43-53. doi: http://dx.doi. org/10.1016/j.jmaa.2010.02.014.

[17] Zhao Z., Rong E., Zhao X. Existence for korteweg-de vries-type equation with delay // Advances in Difference Equations, 2012. Vol. 2012. No. 1. P. 64. doi: http://dx.doi.org/10.1186/ 1687-1847-2012-64.

[18] Зароднюк Т. С., Аникин А. С., Финкельштейн Е. А., Бекларян А. Л., Белоусов Ф. А. Технология решения краевых задач для нелинейных систем функционально-дифференциальных 
уравнений точечного типа // Современные технологии. Системный анализ. Моделирование, 2016. T. 49. № 1. с. 19-26.

[19] Zarodnyuk T. S., Gornov A. Yu., Anikin A. S., Finkelstein E. A. Computational technique for investigating boundary value problems for functional-differential equations of pointwise type // Proc. of the VIII International Conference on Optimization and Applications (OPTIMA-2017), Petrovac, Montenegro, October 2-7. - 2017. URL: http://ceur-ws.org/Vol-1987/paper82. pdf.

[20] Beklaryan L. A., Beklaryan A. L. Traveling waves and functional differential equations of pointwise type. what is common? // Proc. of the VIII International Conference on Optimization and Applications (OPTIMA-2017), Petrovac, Montenegro, October 2-7. - 2017. URL: http: //ceur-ws.org/Vol-1987/paper13.pdf.

[21] Gornov A. Yu., Zarodnyuk T. S., Madzhara T. I., Daneeva A. V., Veyalko I. A. A collection of test multiextremal optimal control problems. - New York: Springer, 2013. P. 257-274.

Поступила в редакиию 21.12.2018 